like to make two points.

(1) The issue we addressed in this experiment was how the transition from non-cooperative monomeric globins to cooperative tetrameric haemoglobin may have taken place in the course of vertebrate haemoglobin evolution. Neither $\alpha$ nor $\beta$ globin shows cooperative oxygen binding even though these proteins are assembled into dimers or tetramers in solution. Cooperativity arises only from assembly into tetramers consisting of two $\alpha$ and two $\beta$ chains. In haemoglobin evolution, did the $\alpha$ and $\beta$ chains diverge after globin had been assembled into cooperative homo-multimers, or did cooperativity arise from the assembly of two globins that had already diverged? The absence of $\mathrm{D}$ helix is the most distinct conserved feature which distinguishes modern $\alpha$ and $\beta$ globins and the principal conclusion of our paper ${ }^{1}$ was that cooperative tetramer can be assembled with or without the D helix. The experiment carried out at one temperature was enough to demonstrate the important conclusion that the deletion of $\mathrm{D}$ helix is likely to have been an independent event from assembly into cooperative tetramers.

(2) The conditions used for measuring oxygen equilibrium curves, which were unfortunately omitted from our original paper, were $50 \mathrm{mM}$ bis-Tris buffer, $\mathrm{pH}$ $7.4,0.1 \mathrm{M} \mathrm{Cl}^{-}, 25^{\circ} \mathrm{C}$. These conditions closely match those found within the red cell and are widely used as standard conditions for this type of experiment. In the case discussed by Giardina et al., the variation with temperature of oxygen affinity is considerably increased by the addition of DPG. This is not surprising as $\mathrm{HbF}$ has changes such as His 143 to Ser at the DPG binding site that weaken DPG binding. The well-characterized DPG-binding site is far removed from the D helix, so it is unlikely that the DPG effect is altered by the D helix. Cooperativity is a sensitive indicator of changes to the allosteric balance between the low-affinity $\mathrm{T}$ state and the high-affinity $R$ state of the protein. Our results show that functionally important $\mathrm{C}$ and $\mathrm{E}$ helices can be connected either by a loop or a small helix without disturbing this fine balance.

KIYOSHI NAGAI NOBORU KOMIYAMA MRC Laboratory of Molecular Biology, Hills Road, Cambridge CB2 $2 \mathrm{QH}$, UK

JEREMY TAME

Department of Chemistry,

University of York

Heslington, York YO1 5DD, UK

1. Komiyama, N. H. et at. Nature 352, 349-351 (1991) 2. Doyle, M. L. et al. Biochem. J. 260. 617-619 (1989) 3. Tomita, S. J. biol. Chem. 256, 9495-9500 (1981)

4. Perutz, M. F. Mechanism of Cooperativity and Allosteric Regulation in Proteins (Cambridge Univ. Press, 1990).

\title{
Dystrophin mRNA in lyophilized tissue
}

SIR - Khurana et al. ${ }^{1}$ have recently shown that biopsy tissue can be preserved through lyophilization for later antibody-based diagnostic testing for Duchenne or Becker muscular dystrophy (DMD/BMD). We have found that lyophilized specimens can also be stored at room temperature for extended periods of time for eventual RNA-based diagnostic testing for this disease.

Cryostat sections $(20-\mu \mathrm{m})$ were cut from three biopsies to yield a total of approximately $25 \mathrm{mg}$ of each tissue. The sections were flash-frozen in liquid nitrogen and lyophilized overnight under constant vacuum as described in ref. 1 . Two of the biopsies were from BMD patients with dystrophin gene deletions previously identified by DNA analysis and the

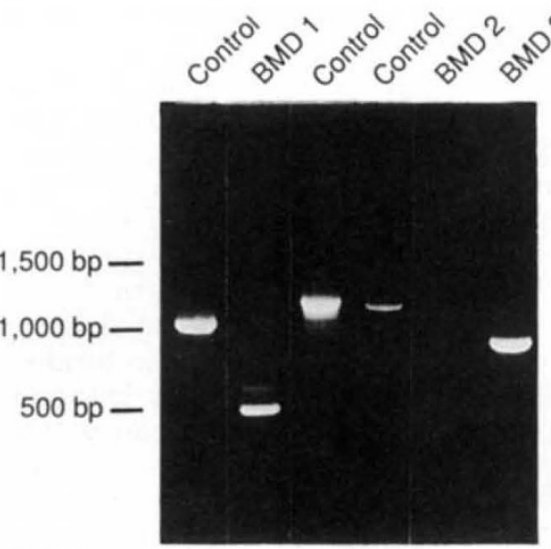

Alterations in RT-PCR products from lyophilized samples. Lane 1, normal sized product amplified from the $5^{\prime}$ end of the dystrophin gene. Lane 2 , reduced-sized product amplified from patient 1 using the same primer set, indicating the absence of exons in this region. Lanes 3 and 4 , normal products from amplification with two overlapping primer sets within the rod region of the dystrophin gene. Using the same two overlapping primer sets, lanes 5 and 6 show the missing product from one primer set and the reduced sized product from the other primer set. indicating a deletion lying somewhere between exons 44 and 52 in the patient's dystrophin gene.

third was obtained from an individual with no dystrophin gene deletion. The lyophilized tissue samples were coded to allow the RNA analysis to be performed blind, with no indication of the sample identity or the deletions present in the patient samples.

$\operatorname{Poly}(\mathrm{A})^{+}$RNA was extracted from the lyophilized samples after 7 days' storage at room temperature. The RNA was reverse transcribed to obtain complementary DNA for polymerase chain reaction (PCR) amplification. Primers used in the PCR amplification were designed as overlapping sets to amplify the entire 14-kb dystrophin sequence (M.S.A. et al., manuscript in preparation; sequences available upon request).
Following two rounds of amplification with nested primers, the PCR products were separated on agarose gels and analysed for differences from the expected pattern (see figure).

One sample was found to have the normal pattern of bands, indicating no deletion in the individual's dystrophin gene. The other two samples had deletions in two different regions. One had a deletion at the amino terminus lying between exons 2 and 7, and the second had exons missing in the rod region of dystrophin lying between exons 44 and 52. Following the RNA analysis, the sample codes were revealed and the results corroborated by comparison with DNA deletion results previously obtained for these individuals. By DNA analysis, exons 3-7 were deleted in the first sample and exons $45-47$ were deleted in the second sample.

The use of RNA reverse transcription and PCR analysis (RT-PCR) provides insight into the location and extent of dystrophin gene deletions in DMD and BMD patients ${ }^{2,3}$. It offers a higher level of resolution than Southern or multiplex DNA analysis and can identify mutations not detectable at the DNA level.

The RT-PCR technique is also applicable in the diagnosis of other genetic diseases. The use of lyophilized tissue samples should increase the availability of this powerful technique. Lyophilization of sample material obviates the need to transport frozen samples for analysis, which is costly, difficult to do from remote areas, and sometimes results in specimen loss because of thawing. Because the lyophilized tissues can be shipped at room temperature, this preservation method gives hospitals and physicians throughout the world access to the few specialized centres offering RNA-based testing techniques.

MARYDILYS S. ANDERSON LOUIS M. KUNKEL

Division of Genetics and

Howard Hughes Medical Institute at Childrens Hospital,

TEVIR S. KHURANA

Program in Neuroscience,

Harvard Medical School,

300 Longwood Avenue, Boston, Massachusetts 02115, USA

1. Khurana, T. S. et al. Lancet 388, 448 (1991)

2. Chelly, J. et al. Nature 333, 858-860 (1988)

3. Roberts, R. G. et al. Lancet 336, 1523-1526 (1990).

\section{Scientific Correspondence}

Scientific Correspondence is intended to provide a forum in which readers may raise points of a scientific character. They need not arise out of anything published in Nature. In any case, priority will be given to letters of fewer than 500 words and five references. 